membranous nephropathy, and Praga et al. have now investigated whether monotherapy with another calcineurin inhibitor-tacrolimus-has a similar effect.

The trial included 48 adults with biopsy-proven membranous nephropathy, preserved renal function, nephrotic-range proteinuria $(>3.5 \mathrm{~g} / 24 \mathrm{~h}$ ) and hypoalbuminemia $(<3 \mathrm{~g} / \mathrm{dl})$ for $\geq 9$ months despite treatment with angiotensin-convertingenzyme inhibitors or angiotensin-receptor blockers. Patients were randomized to a tacrolimus group $(n=25$; starting at $0.05 \mathrm{mg} / \mathrm{kg} /$ day for 12 months followed by a 6-month taperingoff period) or a control group ( $n=23)$. All patients continued on angiotensin-converting-enzyme inhibitors or angiotensin-receptor blockers.

Tacrolimus-treated patients were significantly more likely than controls to achieve either complete remission (CR; $<0.5 \mathrm{~g} / 24 \mathrm{~h}$ proteinuria plus stable renal function) or partial remission (PR; proteinuria $<3.5 \mathrm{~g} / 24 \mathrm{~h}$ and $50 \%$ lower than baseline, plus stable renal function) at 2 months, 6 months, 12 months and 18 months ( $36 \%$ vs $9 \% ; 56 \%$ vs $13 \%, 72 \%$ vs $22 \%$, and $76 \%$ vs $30 \%$, respectively; $P<0.05$ for all). Mean time to $C R$ or PR was significantly shorter in tacrolimus-treated patients than in controls (6.1 months vs 11.3 months; $P=0.003$ ). Although no tacrolimus-treated patients in $\mathrm{CR}$ or PR relapsed during the tapering period, nearly half relapsed after tacrolimus withdrawal. Marked worsening of renal function ( $\geq 50 \%$ serum creatinine increase) was more common in control patients than in tacrolimus-treated patients ( $26 \%$ vs $4 \% ; P<0.03)$. Tacrolimus was well tolerated and no patient withdrew as a result of tacrolimus-related adverse effects.

Original article Praga M et al. (2007) Tacrolimus monotherapy in membranous nephropathy: a randomized controlled trial. Kidney Int 71: 924-930

\section{Factors that might affect risk of gadodiamide-related nephrogenic systemic fibrosis}

Reports have indicated a link between gadolinium-containing MRI contrast agents (particularly gadodiamide) and nephrogenic systemic fibrosis (NSF; formerly known as nephrogenic fibrosing dermopathy) in people with renal failure. Not all renal failure patients exposed to these agents develop NSF, however, indicating the involvement of cofactors.
A recent case-control study included 19 patients (mean age 52 years) with histologically confirmed NSF and 19 age-matched and sexmatched controls with no signs of NSF. All participants had been exposed to gadodiamide while in stage 5 chronic kidney disease (CKD5) at the same hospital. Controls had been exposed to gadodiamide at least 12 months before the study; as NSF develops within 3 months of exposure, this period ensured that controls were unlikely to subsequently develop NSF.

The mean gadodiamide dose received by cases shortly before NSF onset was similar to the mean dose received by controls at their most-recent $\mathrm{MRI}$ examination $(0.29 \mathrm{mmol} / \mathrm{kg}$ vs $0.28 \mathrm{mmol} / \mathrm{kg}$ ). Cumulative gadodiamide exposure while in CKD5, however, was significantly higher in NSF patients than in controls $(0.41 \mathrm{mmol} / \mathrm{kg}$ vs $0.31 \mathrm{mmol} / \mathrm{kg} ; P=0.05)$ and was higher in severe cases than in nonsevere cases $(0.49 \mathrm{mmol} / \mathrm{kg}$ vs $0.33 \mathrm{mmol} / \mathrm{kg} ; P=0.02)$. Compared with controls, patients with NSF had significantly higher serum ionized calcium and phosphate levels ( $P<0.05$ for both), and tended to have been receiving higher epoetin- $\beta$ doses at the time of gadolinium exposure. Severe NSF mainly affected patients who had been having regular hemodialysis at the time of gadodiamide exposure, indicating that loss of residual renal function in patients with CKD5 might increase the risk of severe NSF.

Original article Marckmann P et al. (2007) Case-control study of gadodiamide-related nephrogenic systemic fibrosis. Nephrol Dial Transplant [doi: 10.1093/ndt/gfm261]

\section{Kidneys can be safely harvested from transfusion-refusing living donors}

Despite improvements in surgical techniques, living kidney donors are still at risk for serious complications that might require blood transfusion. Many transplant programs are reluctant to consider people who do not wish to receive human blood products as potential donors. In a recent paper, a group has discussed the issues surrounding the use of such transfusionrefusing (TR) individuals, whose inclusion would expand the pool of living donors.

The authors report on 82 living donor nephrectomies, 8 of which were performed on TR individuals. In TR cases, nephrectomies were performed with two senior surgeons present, 